\title{
Politique
}

Politique

\section{Les minorités en France, au Canada et au Québec : minoritaires ou mineures?}

\section{Julien Bauer}

Numéro 20, automne 1991

URI : https://id.erudit.org/iderudit/040697ar

DOI : https://doi.org/10.7202/040697ar

Aller au sommaire du numéro

Éditeur(s)

Société québécoise de science politique

ISSN

0711-608X (imprimé)

1918-6584 (numérique)

Découvrir la revue

Citer cet article

Bauer, J. (1991). Les minorités en France, au Canada et au Québec :

minoritaires ou mineures? Politique, (20), 5-33.

https://doi.org/10.7202/040697ar
Résumé de l'article

Les minorités dans les États contemporains sont à la fois minoritaires et mineures. L’État libéral, à la française, nie leur existence légale mais les reconnaît de facto. L'État corporatiste, à la canadienne et à la québécoise, répartit la population en trois groupes : les Amérindiens, les peuples fondateurs et les autres. Les premiers jouissent de droits exclusifs, les autres, les minorités, de droits négatifs (non-discrimation et éducation) et, dans certains cas, positifs (programmes d'accès à l'égalité).

La définition des minorités aboutit à des droits réservés avec politiques et administrations spécialisées. Paradoxalement, l'État libéral traite de manière pragmatique les minorités qu'il se refuse de reconnaître alors que l'État corporatiste ne cesse de multiplier les distinctions entre les groupes de la société, marginalisant ainsi les minorités. 


\title{
LES MINORITÉS EN FRANCE, AU CANADA ET AU QUÉBEC : MINORITAIRES OU MINEURES?
}

\author{
Julien Bauer \\ Université du Quêbec ‡ Montréal
}

\begin{abstract}
Les minoritês dans les États contemporains sont à la fois minoritaires et mineures. L'État libéral, a la française, nie leur existence légale mais les reconnaît de facto. L'État corporatiste, à la canadienne et à la québécoise, répartit la population en trois groupes: les Amérindiens, les peuples fondateurs et les autres. Les premiers jouissent de droits exclusifs, les autres, les minorités, de droits négatifs (non-discrimation et éducation) et, dans certains cas, positifs (programmes d'accès à l'égalité).

La définition des minorités aboutit à des droits réservés avec politiques et administrations spécialisées. Paradoxalement, l'État libéral traite de manière pragmatique les minorités qu'il se refuse de reconnaître alors que l'Etat corporatiste ne cesse de multiplier les distinctions entre les groupes de la société, marginalisant ainsi les minorités.
\end{abstract}

L'État-nation, concept relativement récent, prétend rassembler sous la bannière étatique une nation clairement définie. La réalité est différente. Quel que soit leur système institutionnel, État unitaire, fédéral ou confédéral, tous les États contemporains sont confrontés au problème des minorités. Ce problème a d'ailleurs servi d'excuse à toutes les interventions dans les affaires intérieures des États lles Allemands des Sudètes). On le considère souvent comme une mise en question de la souveraineté des Etats. Malgré les réticences des dirigeants politiques, force est de constater que les États, autant ceux dont l'indépendance est récente que ceux dont les frontières sont plus anciennes, $n$ 'arrivent pas a concilier frontières et minorités, et plus particulièrement minorités ethniques.

Le mot même de minorité prête à confusion. On y englobe tous les groupes, y compris les groupes démographiquement majoritaires, c'est-à-dire qui se perçoivent et sont perçus comme présentant des caractéristiques propres que ne partage pas la société officiellement majoritaire : ce sont les 
minorités nationales, ethniques, raciales, religieuses, linguistiques, sexuelles, etc. Dans le présent article, nous ne considérerons que les minorités internationalement reconnues comme telles et les groupes a caractéristiques héréditaires (race, ethnie, religion, langue) par opposition aux groupes a caractéristiques personnelles (sexe, orientation sexuelle, handicap). A cet égard, I'article 27 du Pacte international relatif aux droits civils et politiques stipule que :

"dans les États où il existe des minorités ethniques, religieuses ou linguistiques, les personnes appartenant à ces minorités ne peuvent être privées du droit d'avoir, en commun avec les autres membres de leur groupe, leur propre vie culturelle, de professer et de pratiquer leur propre religion, ou d'employer leur propre langue».

II y a également confusion entre mineur et minoritaire. Le premier sens de minorité est : "l'état d'une personne qui n'a pas encore atteint l'âge où elle est légalement considérée comme pleinement capable et responsable de ses actes»' Un mineur est donc un adulte en puissance, à qui il manque la maturité nécessaire pour prendre certaines décisions. Il est placé sous la tutelle d'un adulte responsable, capable de prendre des décisions bénéfiques pour lui et pour le mineur.

Une minorité est une collectivité particulière au sein de la société, à qui il manque certains attributs que possède la population majoritaire. Pour certaines décisions, la minorité est placée sous la tutelle de l'État, qui a la capacité de prendre des décisions bénéfiques pour toute la société, c'està-dire pour la majorité et les minorités. Comme le remarque Vincent Lemieux, une "minorité "totale" est moins sur le plan numérique, moindre sur le plan socio-culturel, mineure sur le

1. Définition du Robert, 1972, p. 1091. 
plan sociétal, est minorisée sur le plan politiquen ${ }^{2}$. Le glissement du quantitatif au qualitatif paraît difficilement évitable : majorité et minorité dans un État ne sont pas à égalitéc; la majorité est non seulement supérieure en nombre, elle le devient aussi en responsabilité. Une plus grande responsabilité débouche sur un plus grand contrôle de l'État. Majorité devient donc synonyme de «sujet de l'État»; à l'inverse, minorité signifie "objet de l'État».

En dépit du discours officiel, qui a parfois tendance à les nier, les minorités existent. Confronté à ce phénomène, l'État peut lui accorder une reconnaissance de jure ou de facto. Dans cet article, nous présentons le paradoxe de la relation entre l'État et les minorités. L'État libéral a la française nie, au nom des droits individuels, l'existence des minorités; il est cependant forcé de les reconnaître. L'État corporatiste à la canadienne et à la québécoise reconnaît et institutionnalise les minorités, mais le fait souvent au détriment des droits individuels.

\section{État libéral et minorités : le cas français}

Dans la conception de l'État libéral, tous les citoyens sont égaux en droit et en devoir; plus particulièrement, tout citoyen est électeur et éligible. Depuis que le suffrage universel est étendu aux femmes, les seules restrictions sont d'ordre technique : åge minimum, absence de casier judiciaire, etc. L'égalité des citoyens rend les corps intermédiaires inutiles sinon dangereux. L'idéal type de l'État-nation est imposé à la société civile et a toutes ses composantes : interdiction des affirmations religieuses, des langues régionales, des distinctions entre citoyens. L'école républicaine laïque doit former

2. Vincent Lemieux, «Les minorités et l'État : quelques propositions générales», dans Guillaume, Lacroix, Pelletier et Zylberberg, Minorités et État. Presses universitaires de Bordeaux - Presses de I'université Laval, 1986, p. 10.

3. Sur le rôle ambivalent de l'État face aux minorités, cf. Geoff Dench, Minorities in the Open Society : Prisoners of Ambivalence, Londres et New York, Routledge \& Kegan Bul, 1986. 
des citoyens qui obéissent a l'État, représentant de la nation. C'est ainsi que la France républicaine nie l'existence des minorités religieuses ${ }^{4}$, des minorités linguistiques (le français est la seule langue d'enseignement, les autres langues étant reléguées au niveau de patois dépassés). Même les distinctions sociales sont niées (Loi Le Chapelier de juin 1791 interdisant la syndicalisation des ouvriers). S'il a fallu attendre près d'un siècle pour que les ouvriers obtiennent la légalisation des syndicats ${ }^{5}$, les minorités ethniques, religieuses et linguistiques commencent à peine à être reconnues, sinon légalement, du moins sur le plan politique. C'est que la notion même de minorité va a l'encontre des principes libéraux qui ne considèrent que les individus en tant que tels et non leurs allégeances à un groupe quel qu'il soit. Ce refus de reconnaître les minorités est $d^{\prime}$ autant plus marqué que, en France, cela n'a pas été le fait du gouvernement républicain mais du gouvernement de Vichy. Ce dernier a en effet multiplié les législations antisémites dans le but avoué de se mettre au diapason des vainqueurs allemands, en instaurant un racisme étatique de type nazi.

L'idéologie démocratique libérale et les événements historiques n'ont pu empêcher que le principe de non-reconnaissance des minorités soit l'objet de multiples attaques. La première brèche a été ouverte par le Concordat entre le Vatican et l'Allemagne, qui continue à s'appliquer aux territoires d'Alsace et de Lorraine occupés par l'Allemagne de 1871 a 1918. Dans un pays qui valorise la séparation de l'Église et de l'État, les confessions religieuses d'AlsaceLorraine sont reconnues par l'État et y jouissent de privilèges : prêtres rémunérés par l'État, faculté de théologie àl'Université de Strasbourg, etc. Hors d'Alsace-Lorraine, point de salutl

4. \&ll faut refuser tout aux Juifs comme Nation dans le sens de corps constitué et accorder tout aux Juifs comme individus. II faut qu'ils ne fassent dans l'État ni un corps politique, ni un ordre, il faut qu'ils soient individuellement citoyens". Clermont-Tonnerre, Débat à l'Assemblée nationale, 23 décembre 1789 .

5. Loi Waldeck-Rousseau du 21 mars 1884. 
Depuis une génération, la France est aux prises avec le «syndrome minoritaire" ". Divers groupes qui se définissent surtout par l'attachement à une langue autre que le français, l'occitan, le breton, le corse, revendiquent des droits spécifiques. Le cas de la Corse, séparée physiquement du reste de la France et dont une partie de la population présente des traits ethniques et linguistiques particuliers, témoigne de la résistance au changement. Lorsqu'un texte de loi a prévu la reconnaissance du «peuple corse partie du peuple français», l'opposition y a vu une atteinte aux principes libéraux. Le Conseil constitutionnel a jugé la formulation inadmissible. Un groupe qui, dans un autre contexte, serait considéré comme une minorité se voit refuser même la reconnaissance en tant que partie distincte de la nation. Ce que l'on refuse juridiquement aux Corses est en train d'être reconnu de facto aux "vraies" minorités, qui se composent d'immigrants de fraîche date, dont l'ethnie, la religion et la langue sont différentes de celles de la majorité des Français. Le discours des élites politiques et administratives continue à valoriser l'État démocratique libéral devant lequel tous les citoyens sont égaux et, en toute logique, à s'opposer à l'idée même de minorité. Lors d'une étude que nous avons menée en France en 1990, nous avons recueilli auprès de hauts fonctionnaires des déclarations comme celles-ci : «il faut empêcher la formation de minorité" et "le concept de minorité visible est abominable».

La réalité d'une immigration que, contrairement aux imigrations précédentes, la France n'arrive pas à assimiler, a contraint les responsables à créer des politiques et des situations spécifiques pour des minorités de fait. La plus importante de ces institutions est le Fonds d'action sociale (FAS). Créé par ordonnance le 21 décembre 1958, le Fonds d'action sociale pour les travailleurs musulmans en métropole et leurs familles avait pour mission de venir en aide aux

6. Cf. la contribution de Albert Mabileau, "L'État, la société civile et les minorités en France dans Minorités et État, op. cit., p. 21-32. II y fait allusion au "syndrome minoritaire» (p. 26-28) et a la *multiplication des comportements minoritaires» (p. 27). 
travailleurs algériens en France. Cette institution ne soulevait pas de problème de fond dans la mesure où elle s'adressait à une population originaire d'un territoire alors français, l'Algérie, mais qui ne jouissait pas des droits et privileges des citoyens français. Devenus soit citoyens français, soit ressortissants d'un pays étranger, les travailleurs musulmans d'Algérie en France continuaient à poser des problèmes. Le FAS fut donc renouvelé par un décret le 24 avril 1964 sous le nom de Fonds d'action sociale pour les travailleurs étrangers, puis devint le Fonds d'action sociale pour les travailleurs migrants (décret du 14 septembre 1966) et finalement le Fonds d'action sociale pour les travailleurs immigrés et leurs familles (décret du 18 janvier 1983). Le changement de termes est révélateur: prévue d'abord pour les Algériens musulmans, l'institution s'est occupée ensuite des travailleurs étrangers, donc des non-citoyens, puis des travailleurs d'origine étrangère, naturalisés français ou non. La distinction entre citoyens et non-citoyens s'estompe au profit d'une distinction basée sur l'origine nationale. Dès 1966, le FAS se voit confier une mission sociale plus large auprès des "groupes sociaux posant des problèmes d'adaptation comparables à ceux des travailleurs étrangers"'?

Qui sont ces groupes sociaux? On les retrouve dans les Commissions régionales pour l'insertion des populations immigrées, (CRIPI). Ils comprennent d'abord l'ensemble des populations originaires d'Algérie, du Maroc et de la Tunisie, pays colonisés par la France. Puis ils incluent des migrants originaires du reste du monde, donc sans lien particulier avec la France. Toutefois, ils incorporent des citoyens français noirs, originaires des départements et des territoires français d'outre-mer. Une institution créée en 1958 pour répondre a un besoin pressant et spécifique est devenue responsable non seulement des étrangers vivant en France, mais aussi des étrangers devenus citoyens français et même des citoyens français dont la seule distinction, que récuse la législation, est

7. Yahiel, «Le FAS : question de principe», Revue européenne des migrations internationales, vol. IV, $n^{\circ} 1$ et 2, 1988, p. 107-114. 
d'avoir la peau noire. La couleur de la peau devient plus importante que la citoyenneté.

L'État libéral, symbolisé par la France, continue à prétendre que, du point de vue étatique, la distinction entre citoyens et non-citoyens est la seule démarcation légitime entre les individus, persistant ainsi à nier l'existence des minorités. Sous l'appellation de "populations immigrées", il englobe des immigrants récents, de nouveaux citoyens, des citoyens noirs et il établit politiques et institutions pour ce qu'on appelle ailleurs des minorités. Les minorités sont à la fois niées de jure et reconnues de facto.

\section{État corporatiste et minorités :}

\section{Canada et Québec}

L'État corporatiste a une tout autre conception des minorités. Dans l'État libéral, la relation entre l'État et le citoyen est directe, et les corps intermédiaires $n^{\prime}$ ont aucun rôle légal. Dans l'État corporatiste, la relation avec les citoyens se fait a la fois directement et par le biais des corps intermédiaires, lesquels jouent un rôle légal. Chaque citoyen est à la fois un individu et un membre d'une collectivité.

Le Canada fonctionne dans un cadre corporatiste au sein duquel le Québec constitue une collectivité à part et ce, depuis l'union du Haut et du Bas Canada - union fondée sur deux groupes, les Anglais et les Français, et non sur les individus - jusqu'au défunt accord du Lac Meech. La société canadienne comprend deux catégories de citoyens : ceux qui ont des droits exclusifs protégés par la Charte canadienne des droits et libertés, c'est-à-dire les peuples fondateurs et les Amérindiens; ceux qui ont des droits spécifiques, distincts de ceux des peuples fondateurs et pouvant être négatifs ou positifs.

\section{Les peuples fondateurs}

Les peuples canadien-français et canadien-anglais, considérés comme peuples fondateurs, ont des droits exclusifs. Leurs caractéristiques linguistiques et religieuses sont proté- 
gées par la Charte canadienne des droits et libertés ${ }^{\natural}$ et, là où ils sont minoritaires, ils ont des droits non reconnus aux autres minorités. La protection s'exerce par le biais des langues officielles (articles 16 à 21), du droit à l'instruction dans la langue de la minorité (article 23), des écoles séparées (article 29). Ce dernier point représente le recours à la religion pour protéger les peuples fondateurs là où ils constituent des minorités linguistiques.

Pour réduire l'inégalité entre les divers groupes, le gouvernement canadien s'est lancé dans une politique de multiculturalisme. C'est que les peuples non fondateurs revendiquaient leur place au soleil. Dès 1965, le sénateur Paul Yuzik déclarait:

\begin{abstract}
*The third element ethnic groups now numbering approximately five million persons are co-builders of the West and other parts of Canada, along with the British and French Canadians and are just as permanent a part of the Canadian scene [...] As co-founders they should be co-partners who would be guaranteed the right to perpetuate their mother tongues and cultures" ${ }^{9}$.
\end{abstract}

Quelques années plus tard, devant le mécontentement des minorités de l'Ouest face à la Commission royale d'enquête sur le bilinguisme et le biculturalisme, le mandat de la Commission était élargi. II devait

8. La Loi constitutionnelle de 1982 comprend la Charte canadienne des droits et libertés (annexe B, partie I). Ratifiée le 29 mars 1982, la Charte comprend 34 articles, un quart d'entre eux visant spécifiquement les peuples fondateurs.

9. Cité par John Porter, «Ethnic Pluralism dans Canadian Perspective», dans Glazer et Moynihan, ed., Ethnicity, Theory and Experience, Cambridge, Harvard University Press, 1975. 
"faire enquête et rapport sur l'état présent du bilinguisme et du biculturalisme et recommander des mesures à prendre pour que la Confédération canadienne se développe d'après le principe de l'égalité entre les deux peuples qui l'ont fondée, compte tenu de l'apport des autres groupes ethniques a l'enrichissement culturel du Canadą» (c'est nous qui soulignons).

Les deux peuples fondateurs étaient caractérisés par les deux langues officielles, mais le «pluralisme culturel» devenait "l'essence même de l'identité canadienne" ${ }^{10}$.

L'obligation de rassurer les minorités témoignait de la supériorité des peuples fondateurs. Supériorité qui se manifeste dans le traitement réservé aux minorités «officielles», c'est-à-dire aux membres des majorités qui sont démographiquement minoritaires dans les provinces. Au Québec, au cours des vingt dernières années, on a vu une évolution des Canadiens anglais, branche d'un peuple fondateur égal. Ils sont devenus des Anglo-Québécois, minorité reconnue mais ayant des droits inférieurs à ceux de la majorité québécoise (loi 178). Dans le même ordre d'idées, les Canadiens français de l'Ouest reprochent aux politiques multiculturelles de les traiter en minorité et non en branche d'un des peuples fondateurs. Tant les fondateurs, majoritaires ou minoritaires, que les minorités savent que certains droits sont plus protégés que d'autres.

Les peuples fondateurs, même là où ils sont démographiquement minoritaires, voient leurs droits reconnus, du moins théoriquement. Les minorités, même là où elles sont démographiquement majoritaires, restent mineures : leur langue, leur culture et leur religion ne sont pas garanties, et sont tout au plus tolérées dans la mesure où ces minorités participent au grand tout canadien ou québécois. Dans sa déclaration à la Chambre des Communes sur le multiculturalis-

10. Déclaration de P.E. Trudeau, le 8 octobre 1971, a la Chambre des Communes. 
me, P.E. Trudeau n'affirmait-il pas que «le gouvernement $s^{\prime}$ efforcera d'aider tous les groupes culturels canadiens qui ont manifesté le désir et la volonté de développer la capacité de s'accroître et d'ajouter à la vie canadienne [...]" ${ }^{11}$. Les mineurs doivent faire preuve de leur bonne foi; les majeurs ne sont pas soumis à ce test.

Le groupe des Amérindiens, bien qu'ayant des droits exclusifs, est limité sur le plan territorial. Reconnus par la Charte larticle 27), administrés par des lois spécifiques ${ }^{12}$ et ayant signé des accords particuliers (Convention de la Baie James), les Amérindiens sur leurs réserves jouissent de droits exclusifs qui leur permettent d'imposer des droits collectifs aux individus, que ces derniers le souhaitent ou non. Le fait que les Amérindiens n'aient obtenu le droit de vote fédéral que le 27 juin 1969 et qu'ils revendiquent de nos jours l'équivalent d'un palier de gouvernement spécifique montre à quel point le traitement qui leur est fait est aux antipodes du libéralisme politique.

Si les Amérindiens ont des droits délimités territorialement, si les peuples fondateurs ont des droits supérieurs aux autres sans avoir de devoirs particuliers, qu'en est-il des minorités ? Elles forment des groupes auxquels s'appliquent des législations particulières soit pour limiter leurs droits, soit pour leur accorder des droits particuliers.

\section{Minorités et droits négatifs : \\ l'éducation et la non-discrimination}

Les commissions scolaires sont un cas de restriction des droits des peuples non fondateurs. Avant la réorganisation de 1973, qui a instauré l'élection des commissaires à la Commission des écoles catholiques de Montréal et au Protestant School Board of Greater Montreal, la situation était curieuse. Certains commissaires de la CECM étaient nommés par

11. Déclaration de P.E. Trudeau, Chambre des Communes, 8 octobre 1971.

12. Loi amendant la Loi sur les Indiens du 12 juin 1985. 
I'Archevêque de Montréal et d'autres l'étaient par la Ville de Montréal. Du côté protestant, qui regroupait la quasi-totalité des minorités refusées par les écoles catholiques, on commençait à reconnaître les membres des minorités comme des citoyens à part entière. Avant 1965, certaines municipalités (Westmount) accordaient aux Juifs le droit de vote aux commissions scolaires; d'autres le leur refusaient (Hampstead). En 1965, le PSBGM a accordé le droit de vote aux non-protestants dans les municipalités autres que Montréal et admis cinq commissaires juifs. Les 25 commissaires se répartissaient ainsi : dix commissaires de Montréal dont cinq sont nommés par la Ville et cinq par le Gouvernement du Québec; dix protestants élus par les municipalités; et cinq Juifs nommés par le Gouvernement. Des dizaines d'années de revendications de la part de la minorité juive avaient abouti à un résultat hybride : seuls les protestants pouvaient être élus; les Juifs étaient nommés au bon vouloir du Gouvernement. Depuis 1973, tout membre du "panel protestant", qui paye des taxes au PSBGM, peut voter et être élu.

Du côté catholique, le droit de vote est apparemment ouvert a tous ceux qui ont le droit de vote aux élections générales 118 ans révolus, citoyen canadien, au Québec depuis au moins six mois, ni sous curatelle publique ni privé de droits électoraux). Mais, en plus, il faut soit avoir un ou des enfants dans une école de la CECM, soit se déclarer de religion catholique. En ce qui concerne les non-catholiques, on distingue les protestants qui ne peuvent acquérir le droit de vote et les "neutres" (ni catholiques ni protestants) qui ont le choix entre les élections à la CECM et celles au PSBGM. Les candidats, eux, doivent non seulement avoir le droit d'être inscrits sur la liste électorale et être domiciliés sur le territoire de la CECM depuis au moins six mois, mais ils doivent aussi déclarer qu'ils sont éligibles sur la liste catholique. Techniquement, cette dernière condition n'est pas une déclaration de foi catholique, mais en pratique elle élimine les non-catholiques.

Jusqu'en 1973 pour les protestants et encore aujourd'hui pour les catholiques, les peuples fondateurs continuent a jouir 
de droits supérieurs à ceux des minorités et ce, sous le couvert de droits religieux.

Cette fermeture aux minorités se retrouve au Conseil supérieur de l'éducation. Créé en 1964, le CSE a pour mandat d'assurer l'adéquation du système d'enseignement à "l'évolution de la société". Dans le Préambule de la Loi sur le Conseil supérieur de l'éducation ${ }^{13}$, on passe d'affirmations libérales :

"attendu que les parents ont le droit de choisir les institutions qui, selon leur conviction, assurent le mieux le respect des droits de leurs enfants;

attendu que les personnes et les groupes ont le droit de créer des institutions d'enseignement autonomes et, les exigences du bien commun étant sauves, de bénéficier des moyens administratifs et financiers nécessaires à la poursuite de leurs fins»

au corporatisme :

"attendu qu'il importe d'instituer, suivant ces principes, [...J, un Conseil supérieur de l'éducation, auquel seront adjoints un comité catholique, un comité protestant et des commissions chargées de faire à ce Conseil des suggestions relativement d divers secteurs de l'enseignement".

Au nom du libre choix, la loi privilégie deux groupes, les catholiques et les protestants, religions des deux peuples fondateurs. Pour éviter tout malentendu, l'article 2 énumère les qualifications des 24 membres : kau moins seize doivent être de foi catholique, au moins quatre de foi protestante et

13. Rapport Parent, Commission royale d'enquête sur l'enseignement, tome 1, 1963, p. 107. 
au moins un doit n'être ni de foi catholique ni de foi protestanten ${ }^{14}$.

Ce corporatisme est accentué par le mode de sélection des membres des comités catholique et protestant. La moitié des membres du comité catholique sont nommés par l'Assemblée des évêques catholiques du Québec, et l'autre moitié par le Gouvernement (article 16). Les protestants étant répartis en plusieurs Églises, c'est le Gouvernement qui nomme les membres du comité protestant après consultation du milieu (article 17). La boucle est bouclée. Un comité dont l'avis favorable est indispensable pour toutes sortes de décisions affectant l'enseignement public catholique, y compris la reconnaissance des écoles comme catholiques (article 22), est nommé pour moitié par un corps intermédiaire, l'Assemblée des évêques. Cette disposition donne, en pratique, un droit de veto a l'Église catholique, droit que ne possède aucune autre confession religieuse. Une confession est «plus égale» que les autres. Les articles 31 et 32 sont explicites : afin d' «accorder des droits et privilèges à une confession religieuse", les articles 3 et 10 de la Charte des droits et libertés de la personne ${ }^{15}$ et les articles 2 a et 15 de la Charte canadienne des droits et libertés ne s'appliquent pas.

Le Québec possède ainsi un organisme public dont la loi constitutive reconnaît le caractère discriminatoire en faveur de deux groupes religieux, catholiques et protestants, les protestants étant numériquement minoritaires mais recevant un statut privilégié en tant que peuple fondateur. La loi de cet organisme a aussi un caractère méprisant à l'égard des "Nini", c'est-à-dire les personnes qui ne sont ni catholiques ni protestantes.

Pour les minorités, il ne s'agit pas d'exclusion de fait comme celle qu'engendrait des politiques d'embauche discriminatoires tacites et que l'on retrouve dans certaines

14. Chapitre C-60.

15. La Charte des droits et libertess de la personne (Lois refondues du Québec, chapitre $\mathrm{C}$-12) a été sanctionnée par l'Assemblée nationale du Québec, le 27 juin 1975. 
administrations publiques. II s'agit plutôt de distinction légale excluant, par le biais de l'appartenance religieuse, ceux qui ne peuvent se réclamer de l'appartenance aux peuples fondateurs.

Si certaines législations limitent les droits des minorités, d'autres en revanche leur accordent des droits spécifiques. Le premier, qui est négatif, est le droit à la non-discrimination. Il est affirmé par les deux Chartes, canadienne et québécoise, respectivement dans les articles 15.1 et 10 . Il est à noter que la Charte canadienne n'inclut pas la langue comme motif de discrimination. Selon Woehrling, "this could probably be interpreted as indicating that the drafters felt that individual linguistic equality is less important and requires less protection than equality between the two major language groups in Canada» $^{10}$. La Charte québécoise, elle, inclut la langue parmi les innombrables motifs possibles de discrimination. Cependant le recours a la clause nonobstant permet de limiter le droit des langues autres que le français (loi 178).

Dans la Charte canadienne, les cas de discrimination visant les minorités comprennent «la race, l'origine nationale ou ethnique, la couleur, la religion» (article 15, alinéa 1). Dans la Charte québécoise, ce sont «la race, la couleur, la religion, la langue, l'origine ethnique ou nationale" (article 10). En pratique, que signifie ce droit à la non-discrimination? Au Québec, la Charte est appliquée par la Commission des droits de la personne (ce qui est prévu à l'article 57) et, depuis la loi 140 du 22 juin 1989, par le Tribunal des droits de la personne. La Charte contient des attendus extrêmement libéraux : "Considérant que tous les être humains sont égaux en valeur et en dignité et ont droit à une égale protection de la loi.» Elle énonce une conception libérale des droits : «Toute personne est titulaire des libertés fondamentales telles la liberté de conscience, la liberté de religion, la liberté d'opinion, la liberté

16. José Woehrling, *Minority Cultural and Linguistic Rights and Equality Rights in the Canadian Charter of Rights and Freedoms», dans McGill Law Journal $n^{\circ} 31,1985$, p. 50-92, cité dans Elkins, «Facing Our Destiny : Rights and Canadian Distinctivenessw, Revue canadienne de science politique, vol. XXII, $n^{\circ} 4,1989$, p. 703. 
d'expression, la liberté de réunion pacifique et la liberté $d^{d}$ association" ${ }^{17}$. Elle interdit plusieurs formes de discrimination $^{18}$. Mais on y trouve aussi une approche corporatiste : wles personnes appartenant à des minorités ethniques ont le droit de maintenir et de faire progresser leur propre vie culturelle avec les autres membres de leur groupe" ${ }^{19}$.

Cette dernière formulation est plus précise que l'article 27 de la Charte canadienne qui dit: *Toute interprétation de la présente charte doit concorder avec l'objectif de promouvoir le maintien et la valorisation du patrimoine multiculturel des Canadiens". La Charte québécoise reconnaît les minorités par l'intermédiaire des individus qui les composent, en donne une définition limitée à l'ethnicité (en retrait par rapport au Pacte international qui comprend également la religion et la langue), mais envisage ces droits dans le domaine exclusif de la culture sans pour autant utiliser le concept, répandu au Québec, de communautés culturelles.

En pratique, la Commission des droits de la personne du Québec, chargée de l'application de la Charte, reçoit des plaintes émanant d'individus. Des groupes peuvent également porter plainte mais au nom d'individus. La CDP peut donc aider les personnes victimes de discrimination, mais $n$ 'a pas juridiction envers les minorités en tant que telles (sauf en ce qui a trait aux programmes d'accès a l'égalité abordés plus loin). Selon son rapport de 1989, la majorité des plaintes a trait au harcèlement sexuel. Selon des responsables de la CDP, c'est parce que les victimes sont de plus en plus au courant de leurs droits, qu'elles sont capables de présenter des plaintes étayées et qu'elles sont aidées par des groupes de soutien. Les autres plaintes portées devant les tribunau $x^{20}$ sont principalement des cas de discrimination en

17. Article 3.

18. Article 10.

19. Article 43.

20. Commission des droits de la personne, Rapport annuel 1989, 1990. tableau XIV : Cas devant les tribunaux en 1989, p. 31-38. 
matière d'âge, de handicap, etc. Comment expliquer le nombre minime de plaintes pour discrimination quant a la race, à l'ethnie, à la couleur, à la religion ou à la langue? Estce angélisme, absence de discrimination ou attestation du cercle vicieux pauvreté-ignorance-racisme?

Dans le même ordre d'idée, le Protecteur du citoyen, dont le mandat est de vérifier ules plaintes de toute personne, compagnie ou association qui s'estime lésée dans ses droits ou croit avoir été injustement traitée par un ministère ou un organisme du gouvernement du Québecx ${ }^{21}$, se plaint, dans son rapport de 1989-1990, de n'avoir pas eu une collaboration suffisante des autorités publiques pour s'occuper davantage des plaintes émanant "des membres des communautés culturelles et ethniques et des populations autochtones, en vue de faciliter l'intégration des premières et de mieux défendre les droits des secondsi ${ }^{22}$. Là encore, il semble que les plaintes émanent surtout de ceux qui connaissent suffisamment le système pour savoir qu'ils ont des droits et pour trouver l'institution vers laquelle se tourner. Ce n'est pas le cas des éléments les plus faibles et les plus démunis, qui connaissent peu leurs droits et les moyens de les faire respecter. Il est symptomatique que la quasi-totalité des plaintes émises en 1989-1990 aient été exprimées en français, soit 20661 . Une minorité a été faite en anglais (1028), et on en relève seulement 14 dans une autre langue ${ }^{23}$.

Comme la Commission des droits de la personne, les services du Protecteur du citoyen sont plus utilisés par la majorité que par les minorités. Celles-ci bénéficient d'une protection légale contre la discrimination mais $y$ ont peu recours. Les raisons tiennent aux minorités elles-mêmes et sont partiellement encouragées par le Gouvernement. Cependant les minorités, en tant que groupes, connaissent de

21. Le Protecteur du citoyen, Rapport annuel 1989-1990, 1990, p. 121.

22. Idem, p. 20.

23. Idem, tableau 2 : la clientèle du Protecteur du citoyen en 1988-1989 et en 1989-1990, p. 106. 
mieux en mieux les rouages du système et n'hésitent pas a les utiliser ${ }^{24}$. En revanche, les personnes qui ont fait l'objet de discrimination à cause de leur appartenance ethnique, religieuse ou linguistique sont souvent les moins bien outillées pour se défendre. Une personne qui parle peu ou ne parle pas le français et ne connait guère plus l'anglais, et qui est originaire d'un État totalitaire où tout ce qui est public est une menace, est certes moins encline à porter plainte devant un organisme de l'État qu'un étudiant auquel un prêt-bourse a été refusé sans motif valable. De leur côté, les dirigeants du système politico-administratif rêvent d'une situation sans problème. Ils considèrent comme un avantage la limitation des moyens dont disposent les organismes dont le mandat est d'aider les victimes de discrimination, y compris les minoritaires. Elle évite une augmentation des couts; elle minimise la visibilité des problèmes; elle les dégage de leur responsabilité.

\section{Minorités et droits positifs : les programmes d'accès à l'égalité}

Depuis quelques années, le droit à la non-discrimination a connu, au Canada et au Québec, un nouveau développement. II s'agit du droit de corriger les situations discriminatoires a l'égard de certains groupes, dont les minorités, et ce, dans plusieurs domaines, principalement dans l'emploi. Les programmes d'accès à l'égalité sont expressément reconnus par les Chartes canadienne (article 15.2) et québécoise (articles 86 et suivants). Au Québec, un programme d'accès à l'égalité, PAE, «doit être approuvé par la Commission des droits de la personne a moins qu'il ne soit imposé par un tribunal» (article 87) et peut aller jusqu'à fixer des quotas pour les groupes visés.

Ce nouveau droit, qui intéresse directement les minorités, ne peut $s^{\prime}$ appliquer dans un système libéral où l'égalité des

24. Evelyn Kallen, Ethnicity and Human Rights in Canada, Toronto, Gage Publishing Ltd, 1982, chapitre 8 : «Minority Responses : The Politicization of Ethnicity», p. 182-212. 
citoyens est considérée comme axiomatique ${ }^{25}$. Dans un régime corporatiste, il trouve sa justification dans un partage des droits individuels et des droits collectifs. Ceux-ci peuvent n'englober que des segments déterminés de la société et être en fait des droits communautaires ${ }^{26}$. Outre ce débat de fond sur la nature du droit, les PAE soulèvent deux questions essentielles: la définition des groupes qui subissent la discrimination et le rôle des pouvoirs publics.

La définition des groupes qui sont l'objet de discrimination est d'autant plus importante qu'elle crée des droits différents pour les divers membres de la société. De l'appartenance ou non à de tels groupes peuvent dépendre l'obtention ou le refus d'un emploi. Louis Le Borgne, dans une communication prononcée au colloque de Rennes "Les étrangers dans la ville" ${ }^{27}$ a critiqué I'incohérence de ces PAE. Peuvent bénéficier des PAE quatre groupes : les femmes, les communautés culturelles, les handicapés et les autochtones. L'apparition du concept de "communauté culturelle", non prévu par la charte, ne pèche pas par excès de clarté. En effet, qui est membre d'une communauté culturelle? Dans un formulaire d'offre d'emploi de l'Office de recrutement et de sélection du personnel de la fonction publique du Québec, cette question était posée aux candidats : "À quelle communauté culturelle

25. L'État libéral peut cependant avoir recours à des PAE pour corriger une situation qui se continue depuis l'époque prélibérale (comme la discrimination contre les Noirs même après l'abolition de l'esclavage aux États-Unis). Selon G. Dench, les PAE américains ont été instaurés dans un double but : améliorer l'image des États-Unis a l'étranger et assurer au Parti démocrate le vote de la minorité noire. Le moyen a été de cibler les groupes les plus défavorisés (Noirs, Indiens, Hispaniques) assurant ainsi une réaction négative des autres minorités (Irlandais, Juifs, Italiens...) pour la plus grande gloire des bénéficiaires traditionnels du système, les WASP. Cf. Dench, op. cit., p. 142-148.

26. Cf. Elkins, 1989, op. cit.

27. L. Le Borgne, «Le Bureaucrate et le métèque : les programmes de discrimination positive au Québec*, présenté au colloque Les étrangers dans la ville, Rennes, 14-16 décembre 1988, 27 p. 
appartenez-vous? ${ }^{28}$. En 1985, les termes de la question ont été changés pour : "communauté culturelle particulière autre que la majorité francophone et les peuples autochtones amérindiens et inuits» ${ }^{29}$. Avant d'être communautés culturelles, les minorités étaient ethniques. Le Conseil consultatif de l'immigration devait être composé de "Québécois autochtones ou des diverses origines reconnues pour l'intérêt qu'ils portent à l'immigration et de membres de différents groupes ethniques" ${ }^{30}$. Les Québécois autochtones n'étaient pas les Autochtones; la distinction entre Québécois d'origines diverses et membres de groupes ethniques n'était pas explicitée. II a fallu attendre un discours de Camille Laurin, alors ministre d'État au développement culturel, prononcé lors d'une réunion de l'Association des études ethniques du Canada (Québec, 5 novembre 1977), pour apprendre la différence entre les Québécois de "vieille souche» et ceux de "nouvelle souche":

"un groupe ethnique est déterminé par un ensemble de caractéristiques et de traditions dont l'existence et la persistance peuvent être observées parmi les familles et les individus. Une nation est une société globale, une entité sociale complète, avec ses propres caractéristiques, sa propre forme d'organisation et d'opération, sa propre continuité historique, sa tradition légale et politique et un territoire clairement défini” ${ }^{31}$.

28. Office de recrutement et de sélection du personnel de la fonction publique, Gouvernement du Québec, Offre de service, formulaire 82-05.

29. Ministère de la Main-d'ceuvre et de la Sécurité du revenu, Placement étudiant, Demande d'emploi d'êté, inscription 1985, formulaire $n^{\circ} 555(094)$.

30. Ministère de I'Immigration, Rapport 1974-1975, p. 50.

31. Canadian Ethnic Studies - Etudes ethniques du Canada, vol. $X, n^{\circ} 1$, 1978 , p. 5. 
Les choses sont claires : les kethniques» sont des individus et des familles sans existence comme groupes ou communautés; la nation, elle, ressemble étrangement à l'État. Quant à la culture nationale, kelle est la propriété commune de toute la population nationale, y compris les minorités ethniques» ${ }^{32}$. Laurin parlait également de promouvoir «le développement des diverses contributions culturelles et leur intégration nécessaire en un ensemble culturel cohérent accessible a la communauté nationale entières ${ }^{33}$. Du moment qu'ils s'expriment en français, les kethniques» peuvent avoir leur propre culture. Dans un document officiel, Laurin distinguait entre trois types de minorités : «la minorité anglosaxonne, les minorités néo-québécoises et les autochtones ${ }^{34}$. Cette approche ayant été rejetée par les intéressés, la position officielle a évolué. De nos jours, on reconnaît non plus des groupes ethniques, mais des «communautés culturelles" qui se distinguent de la majorité par le fait que leur culture n'est pas canadienne-française catholique ${ }^{35}$. Ces communautés, plus que des individus et des familles, ont en commun une culture dont la définition n'est pas claire. Ces communautés se définissent-elles par la langue, la religion, l'ethnie? La langue joue un rôle majeur. Tout non-francophone est ipso facto membre d'une communauté culturelle mais équivoque : il existe des communautés culturelles de langue française comme les Français. La religion est rarement citée, car si les non-chrétiens sont "culturels", nombre de chrétiens, et plus précisément de catholiques, le sont égale-

32. Ibid., p. 5.

33. Ibid., p. 10.

34. La politique québécoise du développement culturel, Québec, Éditeur officiel du Québec, 1978, vol. I, p. 61-91.

35. Selon la Demande d'emploi d'ette, inscription 1985, op. cit., "Sont considérés comme membres d'une communauté culturelle, les personnes dont la langue maternelle est autre que le français, celles qui sont nées à l'extérieur du Canada ou enfin celles qui appartiennent à une communauté culturelle basée sur le caractère d'ordre ethnique, culturel ou autres.» 
ment : catholiques italiens, haïtiens... L'ethnie semble être la marque distinctive : tous les non-Canadiens français sont des membres des communautés culturelles; aucun des Canadiens français ne l'est. La communauté culturelle semble donc désigner les minorités non issues d'un des peuples fondateurs. Que ces minorités soient composées d'immigrants ou de gens vivant au pays depuis des générations, elles sont automatiquement liées à l'immigration, faisant des membres des communautés culturelles des immigrants permanents et, donc, des citoyens mineurs.

\section{Qui est minoritaire?}

Devant la difficulté de définir clairement les communautés culturelles, la tentation, surtout pour les programmes d'accès a l'égalité, a été forte de trouver une définition simple, sinon simpliste, des "vraies" minorités, celles qui ont besoin de l'intervention des pouvoirs publics pour ne plus être victimes de discrimination. Les deux Chartes évoquant la "couleur" comme motif inadmissible de discrimination, on a cru trouver une solution en inventant le concept de "minorité visible». Dans $L$ 'Égalité, ça presse ${ }^{36}$, nous apprenons que les minorités visibles sont «les non-Blancs qui ne participent pas à part entière à la société canadienne" ${ }^{37}$. Les minorités visibles «englobent les membres des Premières Nations et les Métis, de même que les Canadiens originaires des pays africains et arabes, de la Chine, de I'Inde, du Pakistan, du Japon, de la Corée, de l'Asie du Sud-Est, de l'Amérique latine, des îles du Pacifique, des Antilles et des Philippines» ${ }^{38}$. Cette nomenclature est un remarquable exemple de fouillis politico-administratif. Elle crée plus de problèmes qu'elle n'en résout. Elle

36. Comité spécial sur la participation des minorités visibles a la société canadienne, L'égalité, ca presse, Chambre des Communes, Ottawa, mars 1984, p. 2.

37. Ibid., p. 2.

38. Hoid., p. 2. 
nie la visibilité à certains et l'impose à d'autres. Un Juif hassidique ne fait pas partie des minorités visibles, car il est blanc. Un Canadien d'origine haïtienne ou vietnamienne, qui participe à part entière à la société, devient-il ipso facto invisible? L'aspect loufoque de la visibilité des minorités apparaît clairement quand on se pose diverses questions. Par exemple, un nazi réfugié en Argentine et immigrant au Canada est-il un Latino-Américain visible? Pourquoi un Libanais est-il visible et un Sicilien invisible? L'idée est que tout Blanc fait partie de la majorité et, donc, est invisible ${ }^{39}$.

$\mathrm{Si}$ les conflits entre droits individuels et discrimination positive posent des problemes aigus ${ }^{40}$, si les limites entre les groupes minoritaires restent poreuses, que dire de l'attitude des pouvoirs publics qui doivent non seulement déterminer qui est minoritaire, mais quand un programme d'accès à l'égalité est justifié? Concrètement, un PAE dépend du bon vouloir des autorités, la majorité décidant ce qu'elle accordera à ses diverses minorités.

Le Gouvernement du Québec s'est doté, en 1989, d'un programme d'accès à l'égalité à l'intention des communautés culturelles, c'est-à-dire limité «aux membres des minorités visibles et aux personnes qui sont de langue maternelle autre que le français ou l'anglais" ${ }^{41}$. Toutes les minorités dont la langue maternelle est l'anglais sont exclues à moins d'être "visibles». L'ambiguïté du PAE public et son manque apparent de succès ont conduit Daniel Johnson, président du Conseil du Trésor, l'organisme en charge du PAE de la fonction publique, à annoncer, en juin 1991, son intention

39. Nous proposons aux rédacteurs des textes sur la visibilité des minorités l'expérience suivante : d'une femme née au Chili, aux cheveux noirs, et d'une autre née en Suède, blonde, laquelle est la plus visible rue Sainte-Catherine?

40. Nathan Glazer, Affirmative Discrimination : Ethnic Inequality and Public Policy, New York, Basic Books Inc., 1978.

41. Conseil du Trésor, Service de l'accès a l'égalité, Programme d'accès à l'égalité de la fonction publique Québec pour les membres des communautés culturelles 1990-1994, 1990, p. 8. 
d'ouvrir la fonction publique québécoise aux minorités. C'était reconnaître les piètres résultats du PAE.

D'autres organismes publics ont des programmes plus contraignants. En janvier 1989, la Société des transports de la Communauté urbaine de Montréal a mis sur pied un programme de recrutement en précisant les objectifs numériques à atteindre. Le service de police de la CUM, à son tour, a lancé une campagne de recrutement des minorités. Une campagne aux résultats limités (des $\mathbf{7 9}$ nouvelles recrues assermentées le 30 avril 1991, onze provenaient des minorités), mais remarquable du fait qu'elle avait l'appui de la Fraternité des policiers, jusque là peu ouverte aux minorités. Le 11 avril 1991, c'est la CUM elle-même qui a annoncé un PAE.

La même Charte, la même Commission des droits de la personne, les mêmes problèmes aboutissent à la CUM à une politique d'ouverture envers les minorités et a une porte a peine entrouverte au Gouvernement du Québec. Les programmes d'accès à l'égalité sont d'application difficile: les oppositions entre les minorités elles-mêmes, ethniques, visibles et autres, y sont pour quelque chose. II y a aussi l'amalgame des minorités et autres groupes (femmes et handicapés), les réticences que montre la majorité si les PAE deviennent trop contraignants, et la marge de manœuvre laissée aux autorités publiques. Tout cela fait que ces programmes sont de moins en moins des décisions judiciaires ou quasi judiciaires et de plus en plus le résultat des rapports de force politiques. En fait, la discrimination positive, autorisée par la législation, n'est pas univoque; elle peut tout autant corriger des injustices et rétablir des droits pour les minorités que maintenir celles-ci en situation légale d'infériorité. Le fait de connaître l'appartenance d'un candidat à une minorité peut accroître ses chances d'obtenir un emploi. Mais, au contraire, s'il ne réussit pas du premier coup et que le quota est atteint, ses chances de succès sont reportées indéfiniment.

Les PAE, prévus par la loi, sont l'objet de négociations entre les pouvoirs publics et des minorités ou, plus exactement, des groupes parmi les minorités. Les distinctions entre 
citoyens apportées par les PAE peuvent à court terme être favorables aux minorités. Si ces programmes atteignent leurs objectifs, c'est-à-dire l'absence de discrimination due à l'origine ethnique, religieuse ou linguistique des candidats, ils devraient etre appelés à disparaître. La logique administrative laisse supposer que ces programmes se maintiendront, que les objectifs soient entièrement atteints ou non. L'Etat corporatiste, avec les meilleures intentions et souvent appuyé par certaines minorités, aura contribué à une certaine justification des discriminations entre majorité et minorités, l'appartenance minoritaire servant d'intermédiaire entre l'État et les citoyens.

\section{Minorités et droits réservés :} les politiques et administrations spécialisées

En plus du droit à la non-discrimination et de la discrimination positive, les minorités se voient offrir des droits réservés. Les administrations vouées aux minorités (direction du Multiculturalisme et ministère des Communautés culturelles et de ('Immigration) ont des programmes d'aide limités aux minorités et à ceux qui s'y intéressent. Toutes sortes d'organisations, associations et groupes dont le seul mérite est d'être minoritaires ont un accès particulier à des fonds publics qui leur sont réservés. Ainsi le $\mathrm{MCCl}$ fournit-il des subventions à ces groupes minoritaires. Cette exclusivité a des conséquences contradictoires. D'une part, elle permet a des minorités, largement ignorées par les institutions publiques, de goater aux délices des subventions gouvernementales. D'autre part, ces subventions, passant par un canal privilégié, maintiennent les minorités dans une situation de marginalité ${ }^{42}$. Une culture minoritaire ne relève pas des

42. Cf. Louise Fontaine et Yuki Shiose, «Ni Citoyens, ni Autres : la catégorie politique "communautés culturelles"», dans D. Colas, C. Émeri et J. Zylberberg, Citoyenneté et nationalité, perspectives en France et au Québec, Presses universitaires de France, Paris, 1991. L'immigrant y est décrit comme «à la fois inclus - entre autres linguistiquement - et exclu, ou plutôt subordonné en permanence comme minorité différenciée de la majorité, dans le discours gouvernemental», p. 436 . 
affaires culturelles mais des communautés culturelles. Bien mieux, les droits et libertés des individus, but ultime des Chartes, passent par l'inégalité institutionnalisée. Le programme d'édition et de publication du multiculturalisme canadien est explicite : pour recevoir une aide financière, un projet doit porter sur le multiculturalisme (ce qui laisse la possibilité à des minoritaires et à des majoritaires de faire une demande), mais les requérants doivent aussi «prouver qu'ils ont reçu l'appui moral et financier du groupe ou de la collectivité ethnoculturelle à desservir" ${ }^{43}$.

A nouveau, la minorité sert d'écran entre les individus et l'État. Les droits exclusifs aux minorités ne peuvent s'appliquer que dans le respect des institutions minoritaires reconnues par l'État. Même dans le domaine intellectuel, les droits individuels des minoritaires sont assujettis aux droits collectifs des minorités, lesquels sont accordés et interprétés par la majorité. L'État corporatiste triomphe.

Lorsque l'État corporatiste accorde des droits à des individus membres de groupes minoritaires. II ne le fait pas par le biais d'une loi s'appliquant à tous les citoyens. II le fait par l'intermédiaire de structures minoritaires, structures qu'il renforce et manipule quand elles existent, qu'il cherche a créer quand elles n'existent pas. L'État intervient pour que ces structures soient au service de ses politiques.

La loi sur la fermeture des commerces le dimanche fournit un exemple de manipulation. Indépendamment de son mérite, une telle loi a des conséquences discriminatoires pour les commerçants auxquels leur religion interdit de travailler un jour autre que le dimanche. Ces commerces sont donc fermés deux jours. A la suite de demandes venant de groupes minoritaires, le Ministère a prévu une dérogation pour des petits commerçants. La question de la détermination des demandes recevables s'est alors posée. Par exemple, qui va confirmer que le commerçant demandeur ferme son commerce un autre jour que le dimanche pour des raisons religieuses? La majorité des demandeurs étant juifs, la solution du

43. Secrétariat d'État, Programme du multiculturalisme, Programme d'édition et de publication, mai 1987, Condition n³, p. 9. 
Gouvernement a été d'attribuer à un organisme juif non religieux, le Congrès juif canadien, la responsabilité de garantir le bien-fondé des demandes. Les Adventistes du $7^{*}$ jour, minorité chrétienne qui chôme le samedi, ont do, eux aussi, recevoir l'aval du CJC pour obtenir l'autorisation d'ouvrir le dimanche. Le Congrès juif canadien, à son corps défendant, est devenu le garant de la bonne foi religieuse non seulement de Juifs, mais d'Adventistes. Donc, pour que les membres de certaines minorités ne subissent pas de discrimination, on leur accorde des droits particuliers. L'exercice de ces droits entraîne l'institutionnalisation de structures minoritaires que ne souhaitent même pas les intéressés.

L'État libéral et l'État corporatiste ont des attitudes qui peuvent sembler contradictoires. Même si le premier ne reconnaît pas les minorités, il s'attaque au racisme par le biais d'une panoplie de lois. Bien que le second reconnaisse les minorités, il ne s'efforce pas visiblement de les protéger contre le racisme. Pour leur part, les élus, loin d'apaiser la situation, contribuent à l'exacerber comme en témoignent les exemples suivants.

En décembre 1987, le secrétaire d'État aux Affaires extérieures, Joe Clark, a demandé par écrit aux premiers ministres provinciaux et aux autres ministres de refuser des invitations lancées par trois organisations sikhs, cela pour ne pas avoir l'air de favoriser le terrorisme. Il est possible que quelques Sikhs soient des terroristes. Si tel est le cas, ils doivent être jugés et, en cas de culpabilité, être condamnés conformément aux lois. Dénoncer publiquement comme terroristes des organisations sikhs, sans les accuser devant les tribunaux, ne semble pas le meilleur moyen de "valoriser le patrimoine multiculturel des Canadiens». Autre exemple : au printemps 1988, pas moins de 250 gendarmes en tenue de combat ont étê dépêchés à la réserve indienne de Kahnawake pour faire cesser un trafic de cigarettes hors-taxe. On ne se rappelle pas avoir vu une telle expédition contre d'autres violateurs des lois, par exemple contre les trafiquants de drogue ou les pollueurs. Immédiatement après cet incident, Ricardo Lopez, député fédéral local et coprésident d'un comité sur le multiculturalisme, a déclaré que «les Indiens devraient 
être envoyés au Labrador»"44. Auparavant, devant les critiques adressées à la Sureté du Québec pour son utilisation, jugée abusive, de la force contre des Indiens de Restigouche qui pêchaient le saumon, le ministre québécois Marcel Léger avait déclaré : "Si les Indiens se tiennent debout, ils ne sentent pas quand on leur tire les cheveux." Aucune de ces déclarations n'a soulevé de condamnation de la part des dirigeants politiques.

Les tribunaux eux-mêmes ne semblent pas toujours saisir la portée de leurs décisions. Dans quel autre État démocratique voit-on un tribunal accepter comme expert, dans un cas de racisme, une personne condamnée dans un autre pays démocratique pour incitation à la haine raciale? Nous faisons allusion ici à Faurisson, condamné en France et promu expert au procès Zundel.

Le Conseil de la radio-télévision canadienne, qui se passionne pour le nombre de minutes attribué en ondes aux divers types d'émissions, ne s'exprime pas sur le contenu raciste de plusieurs émissions, diffusées en particulier pendant la crise amérindienne de l'été 1990. On a l'impression que le premier souci des minorités, la lutte contre le racisme, est du ressort d'organismes spécialisés. Les autres organismes semblent suivre l'exemple des élus politiques et $n^{\prime} y$ attacher qu'une importance minime.

\section{Conclusion}

Le paradoxe que représente un État corporatiste, mal outillé pour lutter contre la discrimination à l'endroit des minorités, s'explique peut-être par la place même qu'occupent les minorités dans le système.

Dans la mesure où l'État libéral ne reconnaît pas les minorités, il peut prendre toutes sortes de mesures non rattachées à un cadre juridique clair. La France, à l'encontre de la tradition républicaine libérale, est en train de créer des précédents sans base juridique. Quand il s'agit d'insérer une

44. The Gazette, 4 juin 1988. 
définition du peuple corse dans un texte de loi, cela crée un tolle. Mais le Fonds d'action sociale pour les travailleurs immigrés et leurs familles, créé par ordonnance et décrets et non par législation, établit une distinction entre des catégories de citoyens et passe de la distinction classique citoyen/non citoyen à celle d'établi/nouvel immigrant et même à celle de Blanc/Noir sans que personne n'y trouve à redire. L'approche étant pragmatique et les minorités n'étant pas appelées par leur nom, les mesures prises à leur égard ne soulèvent pas d'opposition.

Dans la mesure où l'État corporatiste reconnaît les groupes - peuples fondateurs, Amérindiens, minorités -, il se condamne à les définir et à déterminer l'appartenance des individus à des collectivités. Le cas extrême est celui de la nouvelle loi sur les Indiens de $1985^{45}$, entre le gouvernement canadien et les Amérindiens, qui abolit les discriminations sexuelles et leurs conséquences sur l'obtention du statut $d^{\prime}$ Indien. Cette loi contient un extraordinaire article qui définit les catégories Indiens, demi-Indiens et non-Indiens et explique les critères d' "indianité" des enfants nés de couples mixtes (Indien et demi-Indien, Indien et non-Indien, demi-Indien et non-Indien). Cette loi, de nature raciste et voulue par les intéressés et les pouvoirs publics, est inévitable si l'on veut reconnaître la spécificité des Amérindiens et leur statut particulier. Une version édulcorée de ces distinctions entre citoyens se trouve dans l'article 23 de la Charte canadienne, qui reconnaît les droits linguistiques héréditaires des peuples fondateurs. Pour tous les autres droits, les définitions varient avec le temps, l'immigration ou la perception des problèmes. La terminologie utilisée par Statistique Canada est révélatrice : au recensement de 1971 existait le groupe ethnique unègre» ${ }^{46}$; en 1981 , il est devenu : "Canadien de race noire»,

45. Projet de loi C-31, Loi amendant la Loi sur les Indiens, 12 juin 1985.

46. Statistique Canada, Dictionnaire des termes du recensement de 1971, décembre 1972. 
"autres Noirs" et "Noirs d'Afrique»"7, et en 1986 : "Noirs antillais», "Noirs américains", "Noirs canadiens», "autres Noirs» et "Noirs africains»" ${ }^{48}$. On y mélange allégrement race et origine gêographique. Nous avons relevé ci-dessus les concepts nébuleux de communautés culturelles et de minorités visibles.

Toutes ces définitions répondent au même besoin : I'attribution de droits différents aux diverses collectivités qui composent la société. Les droits étant différents, il devient indispensable de définir les différents bénéficiaires de ces droits.

L'État libéral ne parle pas des minorités, mais il s'en préoccupe. L'État corporatiste ne cesse de cataloguer ses citoyens et non seulement de maintenir, mais d'approfondir les distinctions entre les autochtones, les peuples fondateurs et les autres. Chaque catégorie a une administration qui lui est propre. Si les peuples fondateurs sont administrés par I'ensemble du système politico-administratif, les Amérindiens, en raison de leur statut légal particulier, ont leur ministère, et les minorités ont leurs ministères protecteurs.

Les groupes minoritaires sont un fait. Leur assigner des institutions spécifiques signifie que les institutions régulières ne correspondent pas à leurs besoins et ne peuvent pas être réformées pour y répondre. Politiques et administrations spécifiques pour les minorités, loin d'aider celles-ci à participer à la vie de la société, en font des marginaux permanents. Quelle que soit la noblesse des sentiments qui les animent ceux qui favorisent les administrations spécifiques, les distinctions qu'établit l'État entre majorité et minorités ne peuvent aboutir qu'à un seul résultat : la création de groupes mineurs.

47. Statistique Canada, Dictionnaire du recensement de 1981, mai 1982.

48. Statistique Canada, Dictionnaire du recensement de 1986, janvier 1987. 